\title{
Integrated Tracking and Focusing Systems of MEMS Optical Pickup Head
}

\author{
Hao-Der Cheng, Sheng-Yi Hsiao, Mingching Wu, and Weileun Fang
}

Department of Power Engineering, National Tsing Hua University, Hsin Chu 300, Taiwan, R.O.C.

\begin{abstract}
This paper presents a novel bidirectional-driven microelectromechanical systems (MEMS) light manipulation stage. The designed device consists of two actuators integrated with a lens to act as both tracking (in-plane) and focusing (out-of-plane) components for optical pickup head. The actuator is driven by thermal, as well as magnetic, means. According to a static-load deflection test, this device can achieve bidirection actuation with output displacement up to $\pm 54.5 \mu \mathrm{m}$. In addition, this MEMS device has a very small form factor and provides an excellent response time and size reduction. It can be employed on the portable optical storage system.
\end{abstract}

Index Terms-Electromagnetic actuator, light manipulation, optical microelectromechanical systems (MEMS), pickup head.

\section{INTRODUCTION}

$\mathbf{O}$ $\mathrm{N}$ the demands of new-generation optical data storage system, a micro-fabricated optical pickup head with smaller volume, faster response speed, and higher positioning precision is required and plays a important role in improving data-storage speed and the data density. Several researches successfully used microelectromechanical systems (MEMS) technology to develop optical storage components [1]-[4]. However, it is a challenge to achieve a large stroke for a miniaturized device, especially in the out-of-plane direction. In this study, a large-stroke stage with 2-D driving directions is proposed. $\mathrm{A} \pm 54.5 \mu \mathrm{m}$ operation range-focusing actuator is integrated with a thermal-driven in-plane V-beam actuator. The electromagnetic-induced force is employed to drive the stage in focusing direction. Moreover, two driving dimensions are decoupled by the mean of the structure stiffness design. The static-load deflection curve and frequency response were measured. In addition, a lens was attached to the stage to mutate the focusing ability, the focused spot tracks, along with drove stage. Preliminary results were conducted to prove the feasibility of the design.

\section{CONCEPT AND PROCESS DESIGN}

\section{A. Actuation Concept}

As illustrated in Fig. 1(a), a novel bidirectional stage for the tracking and focusing of light beams is presented. This MEMS device monolithically integrates a magnetic actuator (focusing) [5], V-beam thermal actuator (tracking) [6], springs, supporting frame, and lens holder. The commercial microlens, as well as

Digital Object Identifier 10.1109/TMAG.2006.888420

Color versions of one or more of the figures in this paper are available online at http://ieeexplore.ieee.org.

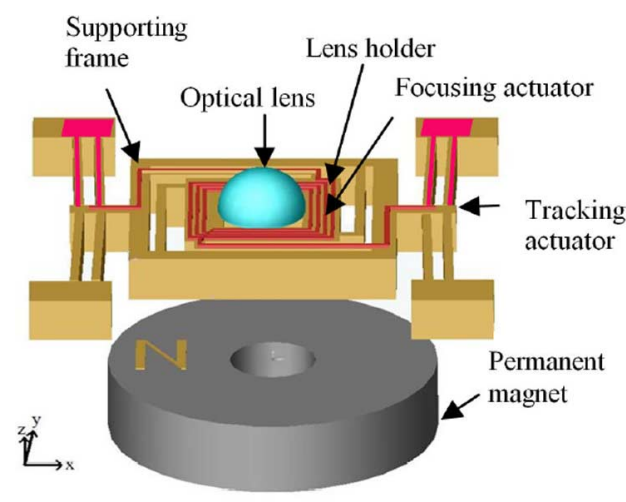

(a)

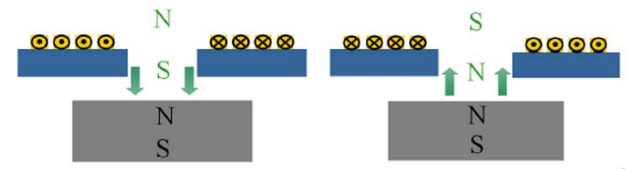

(b)

Fig. 1. (a) Presented tracking and focusing system; underneath the device, an external permanent magnet is used for focusing design. (b) Principle of electromagnetic focusing actuator. By switching the applied current direction, the polarities can be changed to provide either attractive or repulsive force.

the cured UV lens, can be integrated on it. Thus, the incident laser beam can be focused and positioned.

According to joule heating, the V-beam structures experience a thermal expansion so as to push the supporting frame in the in-plane direction. The V-beam actuators located at both sides of the lens holder can move the lens in the opposite direction. In addition, the focusing actuator employs the electromagnetic force to tune the out-of-plane position. The planar coil will induce an electromagnetic field after applying current. The actuator is subjected to an out-of-plane electromagnetic force by the permanent magnet underneath. As indicated in Fig. 1(b), the actuator can move upward or downward by varying the direction of input current, and its displacement is controlled by current magnitude. The present approach allows the actuator to be driven independently in the in-plane and out-of-plane directions. 
(a)

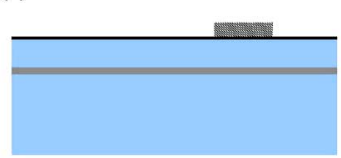

(b)

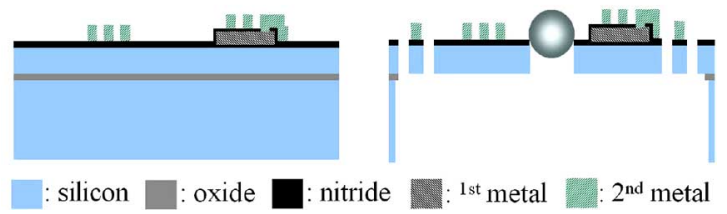

Fig. 2. Fabrication process flow. Five masks total; two metal layers and two isolation layer used.

\section{B. Two Metal SOI Process}

To realize the present actuator, the process steps in Fig. 2 are designed. A SOI wafer with a $20-\mu \mathrm{m}$ device layer, $2-\mu \mathrm{m}$ isolation oxide, and a 350- $\mu \mathrm{m}$ handle layer was used. As shown in Fig. 2(a), the first metal layer was evaporated and patterned after $\mathrm{Si}_{3} \mathrm{~N}_{4}$ was deposited on the SOI wafer. This $1-\mu$ m-thick aluminum metal layer acted as the routing for the planar coil. Underneath, the 5000- $\AA$ low-pressure chemical vapor deposition (LPCVD) low-stress $\mathrm{Si}_{3} \mathrm{~N}_{4}$ acts as isolation between the device layer and the first metal. A second 5000- $\AA$ plasma-enhanced chemical vapor deposition (PECVD) $\mathrm{Si}_{3} \mathrm{~N}_{4}$ was then deposited on the routing and patterned by reactive ion etching (RIE) to define the insulation and via a second metal layer. After that, the second metal layer was then deposited and patterned by liftoff, as in Fig. 2(b). The second metal is $1.5 \mu \mathrm{m}$ thick. The planar coil is mainly formed by the second metal layer. The layers of $\mathrm{Si}_{3} \mathrm{~N}_{4}$ were patterned by RIE and acted as the mask for the following deep reactive ion etching (DRIE) process. Thus, the SOI device layer was patterned, as in Fig. 2(c). Finally, the DRIE was also employed to etch the handle wafer to fully release the devices, as shown in Fig. 2(d). The fully released device was then integrated with a commercial optical lens using a vacuum pickup tool to form the present MEMS optical-positioning stage.

\section{EXPERIMENT RESULT}

The typical process results are shown in Fig. 3. The SEM photo in Fig. 3(a) shows the whole device. The zoom-in photos in Figs. 3(b)-(c) show the electromagnetic focusing actuator and the V-beam thermally tracking actuator, respectively. The bulk silicon material of the handle layer of the SOI wafer is etched; this provides moving space for the stage. Fig. 3(c) shows two metal layers; one metal layer forms a six-turn planar coil, and the other metal layer is simply used for routing. Fig. 3(d) shows a ball lens $300 \mu \mathrm{m}$ in diameter integrated on the stage.

Various experiments have been performed to measure the mechanical characteristics of the actuator, as well as the optical performance. The external permanent magnet flux density is measured to be 0.36 Tesla. The typical measured static-load deflection of the focusing actuator is shown in Fig. 4(a). The actuator has a very good linearity between load and deflection, and it has a $54.5-\mu \mathrm{m}$ displacement when applying a $75-\mathrm{mA}$ current. The in-plane displacement is shown in Fig. 4(b); the stage can (a)

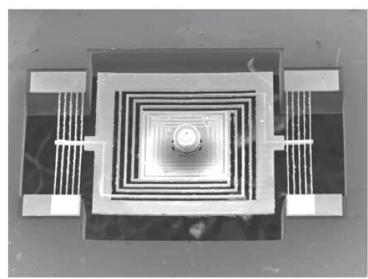

(b)

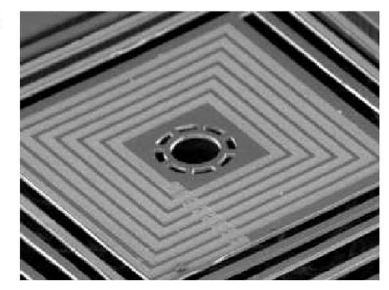

(c)

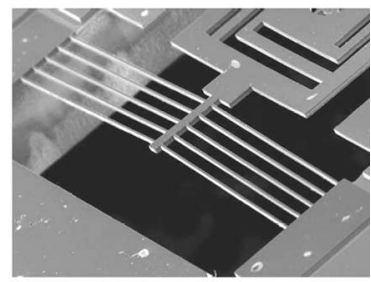

(d)

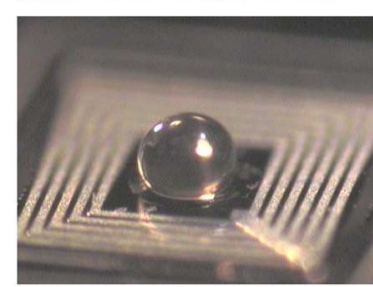

Fig. 3. Fabrication results: (a) the whole device, (b) coil of focusing actuator, (c) V-beam tracking actuator, and (d) integrated ball lens.

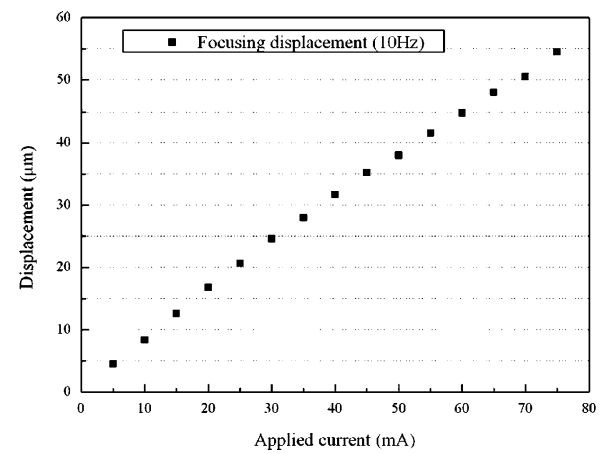

(a)

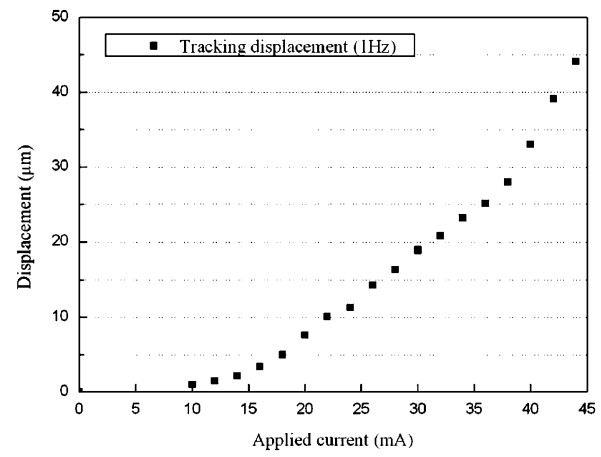

(b)

Fig. 4. Static-load deflection test. (a) Focusing displacement tested under 0.36 Tesla permanent magnet and (b) tracking displacement.

move about $\pm 44 \mu \mathrm{m}$ by driving a thermal actuator with 44-mA current. Fig. 5(a)-(b) shows the frequency response in the focusing and tracking directions. Resonant frequency is measured to be $1.13 \mathrm{KHz}$ in the focusing direction and $2.35 \mathrm{KHz}$ in the tracking direction. The simulation results agree with those in the measurement. Tracking displacement decays quickly as the driving frequency increases in the low-frequency range $(<500$ $\mathrm{Hz}$ ), which results from the characteristic of thermal efficiency.

Finally, the test setup in Fig. 6(a) was established to record the tuning of the light spot using the presented actuator. The laser beam was manipulated to track and focus by means of stage operation. Fig. 6(b) shows the laser spot size tuned by the 


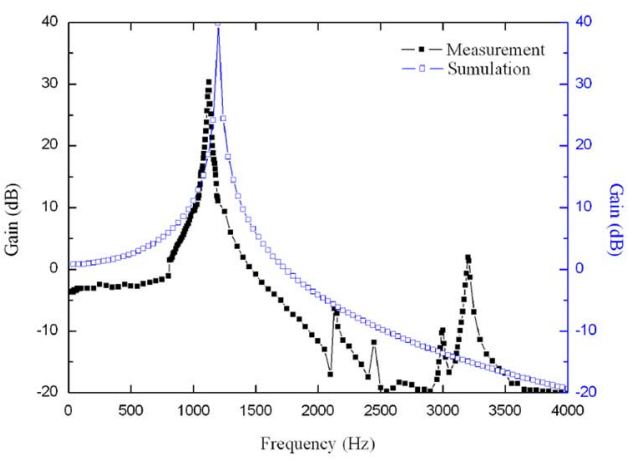

(a)

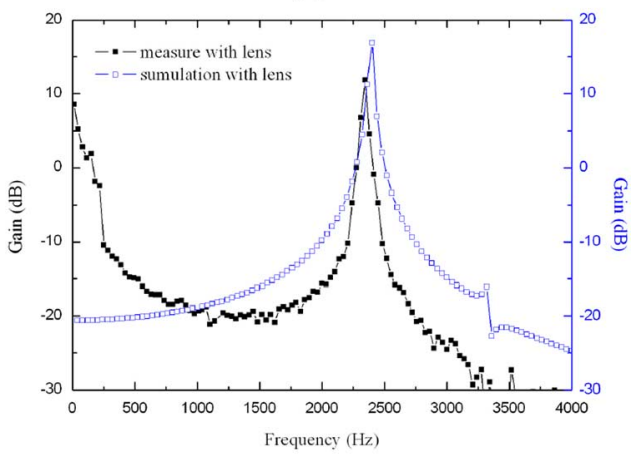

(b)

Fig. 5. Static-load deflection test. (a) Focusing displacement and (b) tracking displacement.

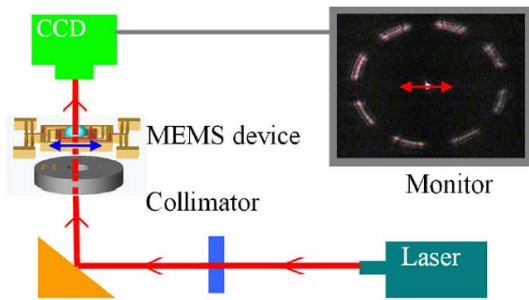

(a)
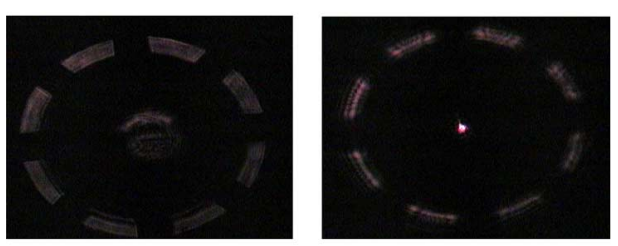

(b)

Fig. 6. (a) Experiment setup for observing manipulated light beam; (b) the laser beam can be focused by operating the stage in the out-of-plane direction.

focusing actuator. Currently, the ball lens focuses the light beam into a $5-\mu \mathrm{m}$ spot.

\section{DisCUSSION AND CONCLUSION}

This study presents a novel actuator to act as both tracking (in-plane) and focusing (out-of-plane) components for optical pickup head. Table I summarizes the specification of the presented bidirection microactuator. A large stroke $Z$-direction movement is achieved. The spring stiffness will be nonlinear
TABLE I

SPECIFICATION OF PRESENTED BIDIRECTIONAL STAGE

\begin{tabular}{|c|c|c|c|}
\hline ITEM & UNIT & FOCUSING & TRACKING \\
\hline Resistance & Ohm & 64.3 & 500 \\
\hline $1_{\text {st }}$ resonant freq. & $\mathrm{KHz}$ & 1.13 & 2.35 \\
\hline DC sensitivity & $\mathrm{mm} / \mathrm{A}$ & 0.727 & 1.00 \\
\hline AC sensitivity & $\mu \mathrm{m} / \mathrm{mA}$ & $0.09(1.5 \mathrm{kHz})$ & $0.024(2.5 \mathrm{kHz})$ \\
\hline Total moving range & $\mu \mathrm{m}$ & \pm 54.5 & \pm 44.1 \\
\hline Moving mass & $\mathrm{mg}$ & $<0.25$ & $<0.4$ \\
\hline Size $(\mathrm{L} \times \mathrm{W} \times \mathrm{T})$ & \multicolumn{4}{|c|}{$<1 \mathrm{~cm} \times 1 \mathrm{~cm} \times 0.05 \mathrm{~cm}$} \\
\hline
\end{tabular}

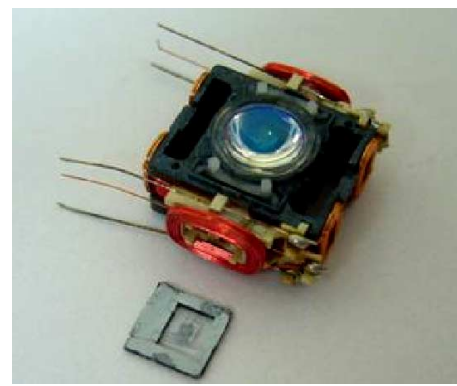

Fig. 7. Comparison photo of the presented pickup head and the commercial component.

with the displacement when it experiences a large deflection. Thus, the nonlinear electromagnetic force can be compensated by the nonlinear spring stiffness, so as to arrive at a linear relation between the driving current and the displacement in Fig. 4(a). In application, a two-metal-layer process was integrated onto the SOI wafer. The device layer acts as structures and SCS thermal actuators, whereas metal routing acts as a electromagnetic coil. The in-plane actuator and out-of-plane actuator are decoupled. The traveling distances in the in-plane and out-of-plane directions are \pm 44.1 and $\pm 54.5 \mu \mathrm{m}$, respectively. The presented miniaturized bidirectional stage has the merits of light weight, small size, and fast response. Fig. 7 is the comparison photo of the conventional voice coil motor pickup head and the presented device. As a result, the MEMS device can act as the key component for a single- or dual-stage optical pickup head for the portable optical storage system.

\section{ACKNOWLEDGMENT}

This work was supported in part by Lite-On IT Corporation.

\section{REFERENCES}

[1] M. C. Wu, L. Y. Lin, S. S. Lee, and K. S. J. Pister, Sens. Actuators A, vol. 50, pp. 127-134, 1995.

[2] Y. Yee, in Proc. MEMS, Miyazaki, Japan, 2000, p. 435.

[3] S. Hata, Y. Yamada, J. Ichihara, and A. Shimokohbe, in Proc. MEMS, Las Vegas, NV, 2002, p. 507.

[4] M. Wu, J. Micromech. Microeng., vol. 16, pp. 1290-1297, 2006.

[5] C. Liu, in Proc. MEMS, Oiso, Japan, 1994, pp. 57-62.

[6] Y. B. Gianchandani, IEEE/ASME J. Microelectromech. Syst., vol. 5, no. 1, pp. 52-58, Jan. 1996.

Manuscript received August 10, 2006 (e-mail: g933765@oz.nthu.edu.tw). 